Brief Report

\title{
Smoke-Isolated Karrikins Stimulated Tanshinones Biosynthesis in Salvia miltiorrhiza through Endogenous Nitric Oxide and Jasmonic Acid
}

\author{
Jie Zhou ${ }^{1}{ }^{1}, \mathrm{Zi}$-xin $\mathrm{Xu}^{1}{ }^{1}$, Hui Sun ${ }^{1}$ and Lan-ping Guo ${ }^{2, *}$ \\ 1 School of Biological Science and Technology, University of Jinan, Jinan 250022, China; \\ zhoujie8761@163.com (J.Z.); aaxzx1231@163.com (Z.-x.X.); sh15854141686@163.com (H.S.) \\ 2 State Key Laboratory of Dao-di Herbs, National Resource Center for Chinese Materia Medica, China \\ Academy of Chinese Medical Sciences, Beijing 100700, China \\ * Correspondence: guolanpingabc@126.com; Tel.: +86-150-9871-1218
}

Received: 5 March 2019; Accepted: 24 March 2019; Published: 29 March 2019

\begin{abstract}
Although smoke-isolated karrikins $\left(\mathrm{KAR}_{1}\right)$ could regulate secondary metabolism in medicinal plants, the signal transduction mechanism has not been reported. This study highlights the influence of $\mathrm{KAR}_{1}$ on tanshinone I (T-I) production in Salvia miltiorrhiza and the involved signal molecules. Results showed $\mathrm{KAR}_{1}$-induced generation of nitric oxide (NO), jasmonic acid (JA) and T-I in S. miltiorrhiza hairy root. $\mathrm{KAR}_{1}$-induced increase of T-I was suppressed by NO-specific scavenger (cPTIO) and NOS inhibitors (PBITU); JA synthesis inhibitor (SHAM) and JA synthesis inhibitor (PrGall), which indicated that NO and JA play essential roles in $\mathrm{KAR}_{1}$-induced T-I. NO inhibitors inhibited $\mathrm{KAR}_{1}$-induced generation of $\mathrm{NO}$ and JA, suggesting $\mathrm{NO}$ was located upstream of JA signal pathway. NO-induced T-I production was inhibited by SHAM and PrGall, implying JA participated in transmitting signal NO to T-I accumulation. In other words, NO mediated the $\mathrm{KAR}_{1}$-induced T-I production through a JA-dependent signaling pathway. The results helped us understand the signal transduction mechanism involved in $\mathrm{KAR}_{1}$-induced T-I production and provided helpful information for the production of $S$. miltiorrhiza hairy root.
\end{abstract}

Keywords: smoke-isolated karrikins; Salvia miltiorrhiza; tanshinone I; jasmonic acid; nitric oxide

\section{Introduction}

Smoke generating from burning plant material has been known to contain karrikins $\left(\mathrm{KAR}_{1}\right)$ —chemicals that are powerful germination promoters. $\mathrm{KAR}_{1}$ plays a major role in natural systems as it is highly active at very low concentrations, shows great potential in agriculture [1] and is promising to be a new plant growth regulator [2-8]. Until now the effects and potential mechanisms of $\mathrm{KAR}_{1}$ on the accumulation of secondary metabolite in medicinal plants has not been reported.

Salvia miltiorrhiza, commonly known as 'Danshen' in Chinese, is one of the most renowned medicinal herbs in China. Its roots and rhizomes have been widely used to remove blood stasis and to eliminate carbuncle throughout Chinese history [9]. In recent years, Danshen has been widely used in medicine, food and cosmetics in European and American markets, which has increased the demand of S. miltiorrhiza [10]. The most important active constituents, tanshinones, are terpenoids. Terpenoids are the largest class of plant secondary metabolites. The biosynthesis of tanshinones can be traced to two distinct routes, the mevalonate pathway (MVA pathway) and the 2-C-methyl-D-erythritol-4-phosphate pathway (MEP pathway), in which a universal five-carbon isoprene precursor, ispentenyldiphosphate (IPP) is used as building block. Tanshinone-type constituents such as tanshinone I (T-I) are considered as major pharmacologically active components and important indexes for measuring the quality of 
Danshen [11,12]. Now, the supply of S. miltiorrhiza to the market mostly relies on field cultivation, so it is vital to take effective measures to improve the content of T-I in the cultivation. In our previous study, it has been found that treatments of plant-derived smoke-water (SW) could markedly increase the content of T-I in S. miltiorrhiza. However, we are not aware of the underlying mechanisms of $\mathrm{KAR}_{1}$ on the accumulation of T-I in S. miltiorrhiza.

The activation of endogenous signaling pathways has been well-documented to play key roles in regulating accumulation of secondary metabolites in plants [13,14]. Signaling molecules, such as nitric oxide (NO), jasmonic acid (JA) and the 'cross-talk' among them have gained great attention [15]. $\mathrm{NO}$ has emerged as a key signal role that exerts various signaling functions in the mechanism of multiple biological functions in plants [6,16-19]. JA plays an essential role in secondary metabolism in medicinal plants [20,21]. However, there is no information describing how these signaling molecules related to the $\mathrm{KAR}_{1}$-induced accumulation of tanshinones in S. miltiorrhiza. The hairy root culture system has been considered as a valuable tool for signal transduction research and a platform for mass production of bioactive components [11,12,22]. Biotic elicitors (yeast extracts), abiotic elicitors (silver ion, La) and plant signal material (JA) have been widely used in enhancing tanshinones production in S. miltiorrhiza hairy root [23-25]. JA increased the accumulation of tanshinone, about 5.8 times that of the control, and also up-regulated the expressions of most investigated genes in S. miltiorrhiza hairy root [26]. JA participated in yeast extracts-induced generation of tanshinones in S. miltiorrhiza [11]. This study aimed to investigate the roles of JA and NO and their 'cross-talk' in $\mathrm{KAR}_{1}$-caused generation of T-I in S. miltiorrhiza, which would help us preliminarily understand the mechanisms involved in $\mathrm{KAR}_{1}$-induced T-I production in S. miltiorrhiza.

\section{Results and Discussion}

\section{1. $K A R_{1}$-Induced Increasing of T-I in S. miltiorrhiza Hairy Root}

S. miltiorrhiza hairy root was treated with and without $\mathrm{KAR}_{1}$ (control) to evaluate the influence of $\mathrm{KAR}_{1}$ on the generation of T-I. The effects of $\mathrm{KAR}_{1}$ on the content of T-I in S. miltiorrhiza were present in Figure 1. Treatment with $\mathrm{KAR}_{1}$ improved the content of T-I $(205.13 \mathrm{mg} / \mathrm{g})$ compared to the control $(176.84 \mathrm{mg} / \mathrm{g})$ at $24 \mathrm{~h}$ after $\mathrm{KAR}_{1}$ treatment. There is little literature on the influence of $\mathrm{KAR}_{1}$ on the production of secondary metabolite in medicinal plants. Aremu et al. [27] reported that treating Tulbaghia ludwigiana with smoke water caused a significant increase in the content of flavonoids compared to the control. Soós et al. [28] demonstrated that smoke water could upregulate the expression of genes and promote biosynthesis of phenolic compounds. Data obtained from this study indicated that $\mathrm{KAR}_{1}$ could enhance the content of T-I in S. miltiorrhiza, which implied that using $\mathrm{KAR}_{1}$ for enhancing the production of tanshinones has significant scientific and industrial implications in hairy root production. 


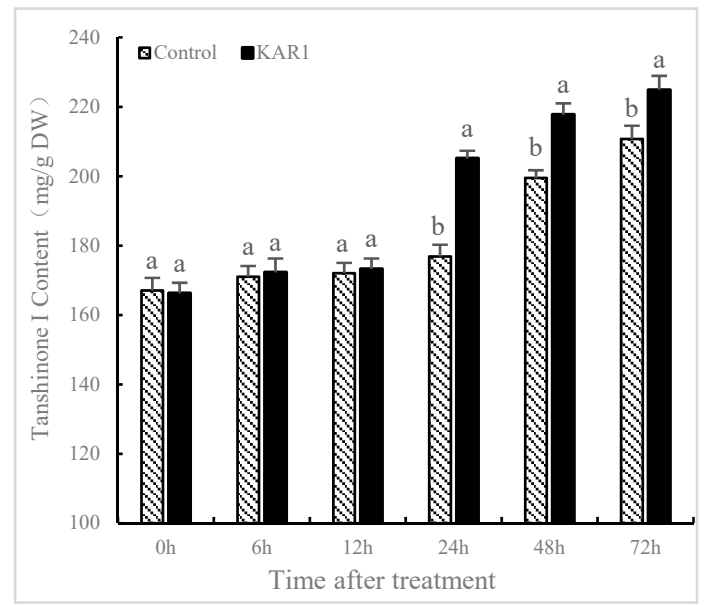

Figure 1. Effects of karrikins on the accumulation of tanshinone I in S. miltiorrhiza hairy root. Data are means of three replicates \pm SD. Different letters indicate significantly different values according to one-way ANOVA followed by Tukey's test $(p<0.05)$.

\subsection{Burst of $N O$ and $J A$ Induced by $K A R_{1}$}

The contents of $\mathrm{NO}$ and JA significantly fluctuated in S. miltiorrhiza treated with $\mathrm{KAR}_{1}$ compared to the control. It has not been found that the levels of NO and JA in the control show significant changes, indicating that the increase of $\mathrm{NO}$ and JA is not owing to development-dependent changes. As shown in Figure 2, NO content was improved significantly with treatment of $\mathrm{KAR}_{1}$, reaching $25.95 \%$ more than the control by $6 \mathrm{~h}(p<0.05), 30.69 \%$ more by $12 \mathrm{~h}(p<0.05)$ and $34.03 \%$ more by $48 \mathrm{~h}$ $(p<0.05)$ respectively. As displayed in Figure 3, JA levels in $\mathrm{KAR}_{1}$-pretreated hairy root displayed a time dependent increase, reaching the peak at 1.41 -fold of control levels at $12 \mathrm{~h}$ after treatment $(p<0.05)$ and then decreased gradually but remained significantly higher $(p<0.05)$ than that of the control. A $\mathrm{KAR}_{1}$-caused burst of JA occurred later than generation of NO. It has been reported that $\mathrm{NO}$ and JA participated in the biosynthesis of matrine, and synergistic action of NO and JA in accumulation of matrine might be in virtue of the mutually amplifying reaction between $\mathrm{NO}$ and JA [29]. Previous studies have shown NO and Put (putrescine) are upstream signals that regulate ginsenoside synthesis during the adventitious roots culture of Panax quinquefolius [10].

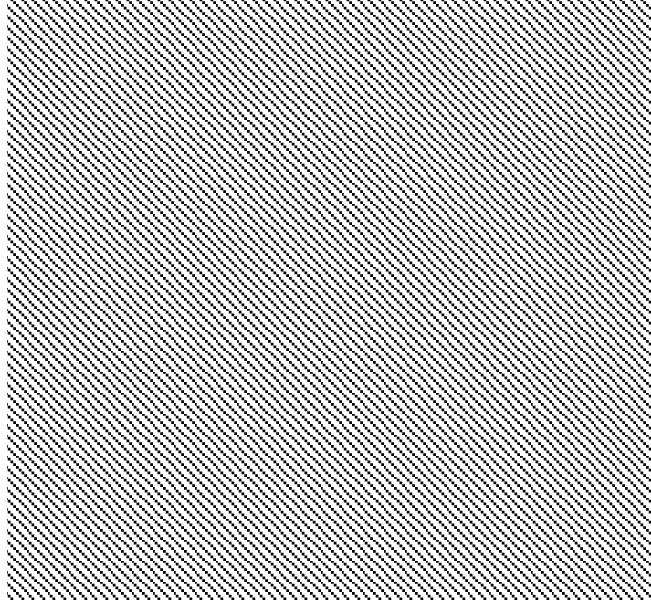

Figure 2. Time courses of $\mathrm{NO}$ level of $S$. miltiorrhiza hairy roots. The roots treated with $\mathrm{KAR}_{1}$ were harvested at determined time points. NO contents of the root were then determined. Data are means of three replicates \pm SD. Different letters indicate significantly different values according to one-way ANOVA followed by Tukey's test $(p<0.05)$. 


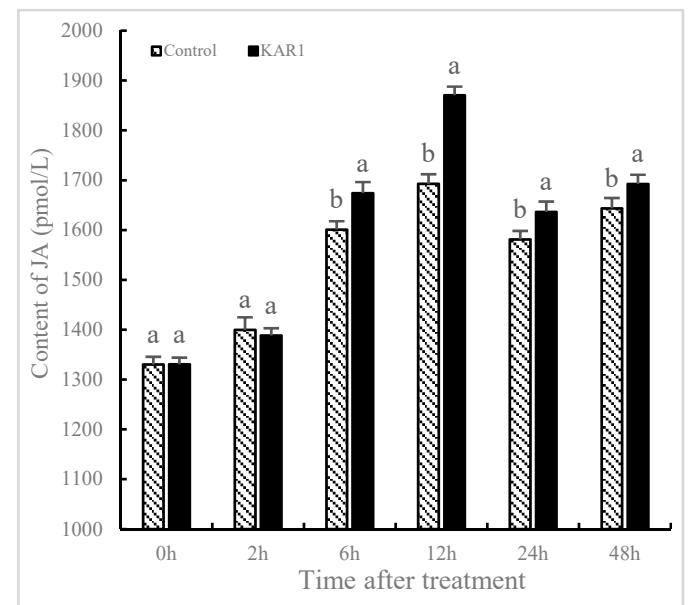

Figure 3. Time courses of jasmonic acid level of $S$. miltiorrhiza hairy root. The roots treated with $\mathrm{KAR}_{1}$ were harvested at determined time points. JA contents of the root were then determined. Data are means of three replicates \pm SD. Different letters indicate significantly different values according to one-way ANOVA followed by Tukey's test $(p<0.05)$.

NO played a pivotal role in the transcriptional regulation of genes related to the phenylpropanoid biosynthetic pathway in Arabidopsis and maize. It improved the expression of transcription factors encoding genes such as ZmP, HY5 and MYB12 and the content of flavonoid [22]. Ren and Dai [16] demonstrated NO-regulated external inducer-induced generation of volatile oil in Atractylodes lancea. It has been investigated that JA acted as a vital signal molecule that regulated secondary metabolism and defense response in plants. Xu et al. [30] identified an important induction effect of JA in heat-shock-induced sesquiterpene production in Aquilaria sinensis. Our results indicated that $\mathrm{KAR}_{1}$-induced generation of $\mathrm{NO}$ and JA occurred earlier than the accumulation of T-I. It is hypothesized that JA and NO may act as signal molecules in $\mathrm{KAR}_{1}$-induced generation of T-I in S. miltiorrhiza. Furthermore $\mathrm{KAR}_{1}$-induced $\mathrm{NO}$ generation occurred earlier than JA.

\subsection{JA Acted as a Downstream Signal of NO Pathway Induced by $K A R_{1}$}

Although a burst of the two signal molecules suggests defensive reactions of the hairy root in response to $\mathrm{KAR}_{1}$, it is still uncertain about their possible upstream and downstream relationships. Thus, the influence of PBITU and cPITO on $\mathrm{KAR}_{1}$-caused JA generation and SHAM and PrGall on $\mathrm{KAR}_{1}$-induced $\mathrm{NO}$ have been investigated. Our test displayed that cPITO and PBITU significantly inhibited the burst of JA induced by KAR $(p<0.05$, Figure 4$)$, however, SHAM and PrGall have not been found to severely affect the generation of $\mathrm{NO}$ (Figure 5). It is not difficult to see that $\mathrm{KAR}_{1}$-induced $\mathrm{NO}$ generation located in upstream of JA biosynthesis. 


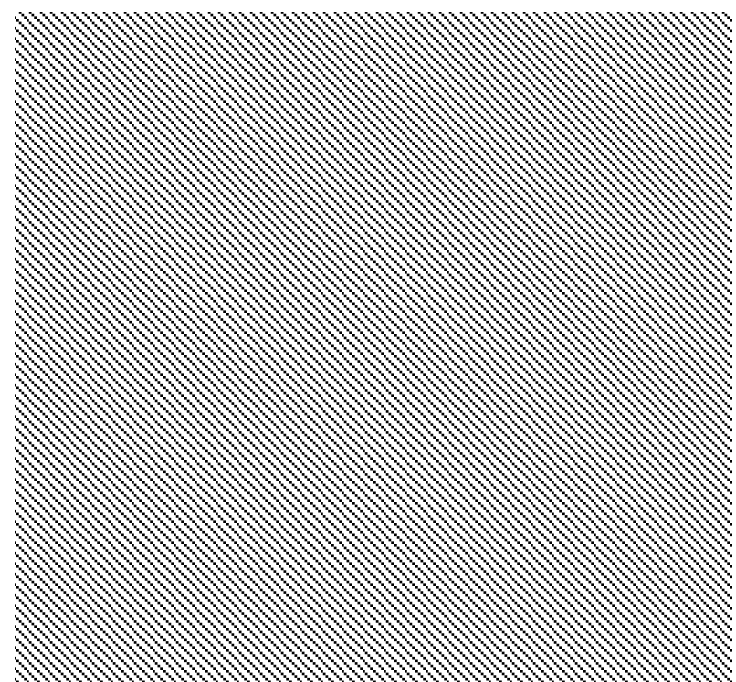

Figure 4. Effects of inhibitors on $\mathrm{KAR}_{1}$-induced JA accumulation in S. miltiorrhiza hairy root. S. miltiorrhiza hairy root treated with $\mathrm{KAR}_{1}$, and various inhibitors were harvested at $12 \mathrm{~h}$ after $\mathrm{KAR}_{1}$ and $\mathrm{NO}$ contents were determined. Inhibitors were pretreated $1 \mathrm{~h}$ before treatment of $\mathrm{KAR}_{1}$. The control received vehicle solvent only. Data are means of three replicates $\pm \mathrm{SD}$. Different letters indicate significantly different values according to one-way ANOVA followed by Tukey's test $(p<0.05)$.

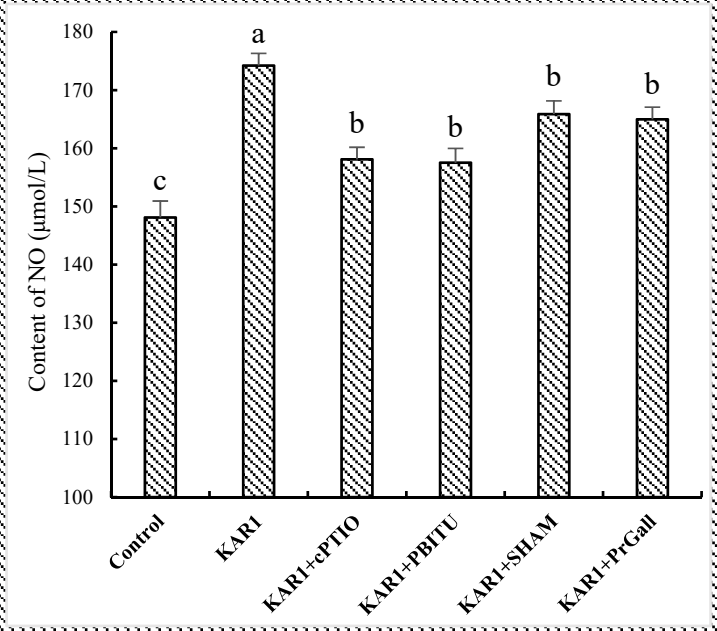

Figure 5. Effects of inhibitors on $\mathrm{KAR}_{1}$-induced $\mathrm{NO}$ generation in S. miltiorrhiza hairy root. S. miltiorrhiza hairy root treated with $\mathrm{KAR}_{1}$, and various inhibitors were harvested at $12 \mathrm{~h}$ after $\mathrm{KAR}_{1}$ and JA contents were determined. Inhibitors were pretreated $1 \mathrm{~h}$ before treatment of $\mathrm{KAR}_{1}$. The control received vehicle solvent only. Data are means of three replicates $\pm \mathrm{SD}$. Different letters indicate significantly different values according to one-way ANOVA followed by Tukey's test $(p<0.05)$.

\subsection{Dependence of $K A R_{1}$-Stimulated T-I Production on NO Accumulation as well as JA production}

It has been exhibited in our experiments that NO generation and production of JA were early

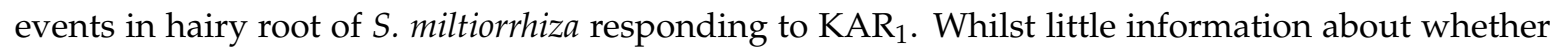
$\mathrm{NO}$ and JA participated in $\mathrm{KAR}_{1}$-induced accumulation of T-I has been known. So we investigated the influence of scavengers and inhibitors of JA and $\mathrm{NO}$ on production of T-I induced by $\mathrm{KAR}_{1}$. As displayed in Figure 6, cPTIO and PBITU significantly $(p<0.05)$ suppressed the increase of T-I induced by $\mathrm{KAR}_{1}$, suggesting that $\mathrm{KAR}_{1}$-induced accumulation of T-I through $\mathrm{NO}$ pathway. Treatments of SHAM and PrGall induced a decline in T-I level, indicating that JA plays a signal part in $\mathrm{KAR}_{1}$-induced increase of T-I. These results were verified by the finding that the suppression of inhibitors of JA and $\mathrm{NO}$ on increase of T-I induced by $\mathrm{KAR}_{1}$ were turned back by treatments of JAMe and SNP (Figure 4). Treatments of NO donor SNP significantly improved the content of T-I, which was evaluated as much 
as $92.70 \%$ of that of $\mathrm{KAR}_{1}$ response. SNP-stimulated increasing of T-I was significantly suppressed by SHAM and PrGall ( $p<0.05)$. Production of T-I in S. miltiorrhiza was stimulated by treatment of JAMe, and it has not been inhibited by PBITU or CPITO. These results displayed that NO-triggered T-I generation depend on JA pathway. This conclusion was further supported by the finding that suppression of SHAM and PrGall on SNP-induced T-I production is relieved by treatment of JAMe (Figure 6).

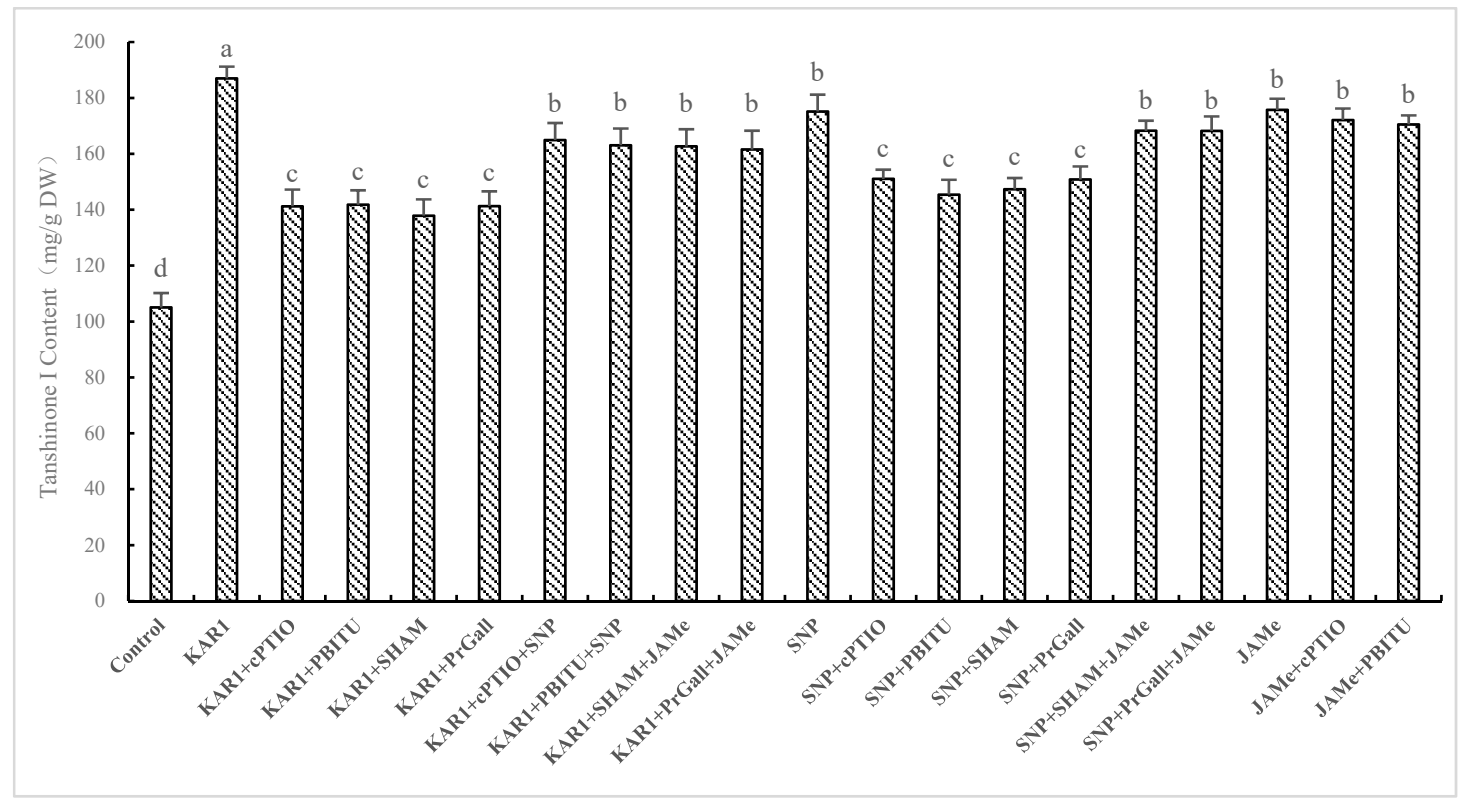

Figure 6. Effects of inhibitors on $\mathrm{KAR}_{1}$-induced T-I production of $S$. miltiorrhiza hairy root. The root treated with $\mathrm{KAR}_{1}$ of, and inhibitors were harvested at $24 \mathrm{~h}$ after $\mathrm{KAR}_{1}$ and T-I production was then determined. Inhibitors were pretreated $1 \mathrm{~h}$ before $\mathrm{KAR}_{1}$. The control received vehicle solvent only. Data are means of three replicates $\pm \mathrm{SD}$. Different letters indicate significantly different values according to one-way ANOVA followed by Tukey's test $(p<0.05)$.

\section{Materials and Methods}

\subsection{Hairy Root Culture and Experimental Design}

S. miltiorrhiza hairy root culture was established by infecting the leaf with Agrobacterium rhizogenes bacterium (ACCC10060). It was incubated in 6, 7-V medium, which contained sucrose of $30 \mathrm{~g} / \mathrm{L}$. Experiments in this study were carried out in $250-\mathrm{mL}$ flasks on an orbital shaker running at $120 \mathrm{rpm}$ and $25^{\circ} \mathrm{C}$ in the dark [31].

After 18 days of culture, $\mathrm{KAR}_{1}$, signal molecular and scavengers were added into the medium and the samples were then allowed to continue culturing for additional days. The content of tanshinone I was determined until sampling for evaluation at adaptation time point. No elicitors were added to the control cultures. Chemical reagents used in the experiment were bought from Sigma Co. (St. Louis, MO, USA), including NO donor sodium nitroprusside (SNP), NO-specific scavenger 2-(4-Carboxyphenyl)-4,4,5,5-tetramethylimidazoline-1-oxyl-3-oxide (cPTIO), nitric oxide synthase (NOS) inhibitors, S,S'-1,3-phenylene-bis(1,2-ethanediyl)-bis-isothiourea (PBITU). Jasmonic acid methyl ester (JAMe), JA synthesis inhibitor salicylhydroxamic acid (SHAM) and JA synthesis inhibitor n-propylgallate (PrGall). Chemical reagent, which was dissolved in water or $0.2 \%$ dimethyl sulfoxide solution, was used in hair roots $36 \mathrm{~h}$ before treatments of $\mathrm{KAR}_{1}$-or signal molecules. Each treatment consisted of 10 replicates, and all treatments were repeated three times. 


\subsection{Preparation of $\mathrm{KAR}_{1}$ Solution}

Smoke water was obtained with the method described by Light et al. [32]. Briefly, dry branches of Crataegus pinnatifida and Magnolia denudata were burned slowly with smoke but no flame, and the smoke was taken through $500 \mathrm{~mL}$ distilled water for $45 \mathrm{~min}$. $\mathrm{KAR}_{1}$ was isolated and identified from smoke water with the method of Van Staden et al. [3] and $10^{-9} \mathrm{M}$ was used in the experiment.

\subsection{HPLC Analysis of T-I}

The content of T-I in hairy root of S. miltiorrhiza was analyzed based on the methods of Liang et al. [33]. An oven-dried sample (0.2 g) was pulverized with a mortar and pestle, and extracted with $20 \mathrm{~mL} \mathrm{70 \%} \mathrm{methanol} \mathrm{under} \mathrm{ultrasonic} \mathrm{treatment} \mathrm{for} 1 \mathrm{~h}$. The resulting mixture was centrifuged at $8000 \mathrm{r} / \mathrm{min}$ for $20 \mathrm{~min}$ and filtered through a $0.22 \mu \mathrm{m}$ syringe filters before high performance liquid chromatography (HPLC) analysis. The content of T-I was analyzed by HPLC on Agilent-1260 apparatus equipped (Palo Alto, CA, USA) with a C18 column $(4.6 \mathrm{~mm} \times 250 \mathrm{~mm}, 5 \mu \mathrm{m}$ particle size), and the flow rate was $1 \mathrm{~mL} / \mathrm{min}$ with the detection wavelength at $275 \mathrm{~nm}$. The working temperature of column was kept at $30{ }^{\circ} \mathrm{C}$ and the sample injection volume was $20 \mu \mathrm{L}$. Separation was achieved by elution using a linear gradient with solvent-B (acetonitrile) and solvent-A (0.2\%-methanoic acid-ammonium). The gradient was as follows: 0-20 min, 20-40\% B; 20-21 min, 40-80\% B; 21-40 min, 80-90\% B; 40-45 min, 90-20\% B.

\subsection{Determination of $\mathrm{NO}$}

The content of NO was estimated in S. miltiorrhiza hairy root using the method of Li et al. [34] with slight modification. According to the principle of the conversion of oxyhemoglobin $\left(\mathrm{HbO}_{2}\right)$ to methemoglobin $(\mathrm{MetHb})$, the content of $\mathrm{NO}$ was determined by spectrophotometry (Shanghai Spectrum Instrument Co. Ltd., China) at 401 and $421 \mathrm{~nm}$. NO accumulation in hairy root was labeled with a specific fluorescent probe of DAF-2DA (4-amino-5-methylamino-2', $7^{\prime}$-difluorofluorescein diacetate).

\subsection{Measurement of $J A$}

The content of JA in S. miltiorrhiza hairy root was determined by the method described in the instruction manual of kit (Shanghai Enzyme Biotechnology Co., Ltd., Shanghai, China). Briefly, $4.0 \mathrm{~mL}$ of phosphate buffer was added to $1.0 \mathrm{~g}$ of hairy root; the mixture was uniformly ground in a mortar on an ice plate; and the homogenate was centrifuged at $2800 \mathrm{r} / \mathrm{min}$ for $20 \mathrm{~min}$ at $4{ }^{\circ} \mathrm{C}$. The supernatant was obtained for the JA content assays. The absorbance was read at $450 \mathrm{~nm}$.

\subsection{Statistical analysis}

ANOVA with SPSS software (version 18.0, SPSS, Inc., Chicago, IL, USA) was used analyze all data and statistical differences among treatments was based on one-way analysis of variance (ANOVA) and a significant difference was concluded at a level of $p<0.05$.

\section{Conclusions}

In summary, the results from this work revealed $\mathrm{KAR}_{1}$ improved the production of $\mathrm{T}-\mathrm{I}$ by triggering the biosynthesis of endogenous NO and JA in hairy root of Salvia miltiorrhiza. Furthermore, $\mathrm{NO}$ regulates the $\mathrm{KAR}_{1}$-induced T-I production through a JA-dependent signaling pathway. Together, the results suggest that $\mathrm{KAR}_{1}$ may be used as a new practical approach to improve the T-I accumulation in S. miltiorrhiza by modulating NO and JA levels. This information will help us better understand the underlying mechanism of $\mathrm{KAR}_{1}$-regulating secondary metabolism. Furthermore, it also suggests strategies to improve the quality of medicinal herbs. Whether these are other downstream molecules participating in JA signal transduction leading to increase of T-I in Salvia miltiorrhiza and their relationships with JA still remains unrevealed. Therefore, it is apparent that we are only at the 
early stage in understanding the signal transduction mechanism in S. miltiorrhiza. Moreover, this means that molecular biology would be used to provide molecular evidence for revealing the signal transduction mechanism in $\mathrm{KAR}_{1}$-regulated secondary metabolism in medicinal plants.

Author Contributions: Methodology, J.Z., Z.-x.X. and H.S.; investigation, Z.J.; resources, L.-p.G.; data curation, Z.-x.X. and H.S.; writing-original draft preparation, J.Z.; writing-review and editing, J.Z. and L.-p.G.; supervision, L.-p.G.; project administration, J.Z.; funding acquisition, L.-p.G.

Funding: This work was financially supported by the Natural Science Foundation of China (NO. 81673527), the National Key Research and Development Project (NO. 2017YFC1702702, 2017YFC1700705), the Shandong provincial Key Research Project (NO. 2017GSF19115, 2017CXGC1303), and the Construction Project for Sustainable Utilization of Valuable Traditional Chinese Medicine Resources (NO. 2060302).

Conflicts of Interest: The authors declare no conflicts of interest.

\section{References}

1. Zhou, J.; Van Staden, J.; Guo, L.P.; Huang, L.Q. Smoke-water improves shoot growth and indigo accumulation in shoots of Isatis indigotica seedlings. S. Afr. J. Bot. 2011, 77, 787-789. [CrossRef]

2. Flematti, G.R.; Ghisalberti, E.L.; Dixon, K.W.; Trengove, R.D. A compound from smoke that promotes seed germination. Science 2004, 305, 977. [CrossRef]

3. Van Staden, J.; Jäger, A.K.; Light, M.E.; Burger, B.V.; Brown, N.A.C.; Thomas, T.H. Isolation of the major germination cue from plant-derived smoke. S. Afr. J. Bot. 2004, 70, 654-659. [CrossRef]

4. Krawczyk, E.; Koprowski, M.; Cembrowska-lech, D.; Wójcik, A.; Kępczyński, J. Synthesis of tricyclic butenolides and comparison their effects with known smoke-butenolide, KAR . J. Plant Physiol. 2017, 215, 91-99. [CrossRef]

5. López-Ráez, J.A.; Shirasu, K.; Foo, E. Strigolactones in Plant Interactions with Beneficial and Detrimental Organisms: The Yin and Yang. Trends Plant Sci. 2017, 22, 527-537. [CrossRef]

6. Salomon, M.V.; Piccoli, P.; Funes, I.P.; Stirk, W.A.; Kulkarni, M.; Van staden, J.; Bottini, R. Bacteria and smoke-water extract improve growth and induce the synthesis of volatile defense mechanisms in Vitis vinifera L. Plant Physiol. Biochem. 2017, 120, 1-9. [CrossRef] [PubMed]

7. Martinez-Baniela, M.; Carlon, L.; Diaz, T.E.; Bueno, A.; Fernandez-Pascual, E. Plant-derived smoke and temperature effects on seed germination of five Helianthemum (Cistaceae). Flora 2016, 223, 56-61. [CrossRef]

8. Morffy, N.; Faure, L.; Nelson, D.C. Smoke and Hormone Mirrors: Action and Evolution of Karrikin and Strigolactone Signaling. Trends Genet. 2016, 32, 176-188. [CrossRef] [PubMed]

9. China Pharmacopoeia Committee. Pharmacopoeia of Peoples Republic of China; China Medical Science and Technology Press: Beijing, China, 2015; p. 76.

10. Yu, Y.; Zhang, W.B.; Li, X.Y.; Piao, X.C.; Jiang, J.; Lian, M.L. Pathogenic fungal elicitors enhance ginsenoside biosynthesis of adventitious roots in Panax quinquefolius during bioreactor culture. Ind. Crop Prod. 2016, 94, 729-735. [CrossRef]

11. Wang, Y.J.; Shen, Y.; Shen, Z.; Zhao, L.; Ning, D.L.; Jiang, C.; Zhao, R.; Huang, L.Q. Comparative proteomic analysis of the response to silver ions and yeast extract in salvia miltiorrhiza hairy root cultures. Plant Physiol. Biochem. 2016, 107, 364-373. [CrossRef]

12. Wu, C.F.; Bohnert, S.; Thines, E.; Efferth, T. Cytotoxicity of salvia miltiorrhiza against multidrug-resistant cancer cells. Am. J. Chin. Me. 2016, 44, 871-894. [CrossRef]

13. Bari, R.; Jones, J.D.G. Role of plant hormones in plant defence responses. Plant Mol. Biol. 2009, 69, 473-488. [CrossRef]

14. Lu, X.J.; Zhang, X.L.; Mei, M.; Liu, G.L.; Ma, B.B. Proteomic analysis of Magnolia sieboldii K. Koch seed germination. J. Proteomics. 2016, 133, 76-85. [CrossRef]

15. Cembrowska-lech, D.; Koprowski, M.; Kępczyński, J. Germination induction of dormant Avena fatua caryopses by $\mathrm{KAR}_{1}$ and $\mathrm{GA}_{3}$ involving the control of reactive oxygen species $\left(\mathrm{H}_{2} \mathrm{O}_{2}\right.$ and $\left.\mathrm{O}_{2}{ }^{\bullet-}\right)$ and enzymatic antioxidants (superoxide dismutase and catalase) both in the embryo and the aleurone layers. J. Plant Physiol. 2015, 176, 169-179. [CrossRef] 
16. Ren, C.G.; Dai, C.C. Nitric oxide and brassinosteroids mediated fungal endophyte-lnduced volatile oil production through protein phosphorylation pathways in Atractylodes lancea plantlets. J. Integr. Plant Biol. 2013, 55, 1136-1146. [CrossRef] [PubMed]

17. Ruan, J.Z.; Li, M.Y.; Jin, H.H.; Sun, L.N.; Zhu, Y.; Xu, M.J.; Dong, J.F. UV-B irradiation alleviates the deterioration of cold-stored mangoes by enhancing endogenous nitric oxide levels. Food Chem. 2015, 169, 417-423. [CrossRef] [PubMed]

18. Ni, J.; Dong, L.X.; Jiang, Z.F.; Yang, X.L.; Sun, Z.H.; Li, J.X.; Wu, Y.H.; Xu, M.J. Salicylic acid-induced flavonoid accumulation in Ginkgo biloba leaves is dependent on red and far-red light. Ind. Crop Prod. 2018, 118, 102-110. [CrossRef]

19. Xu, M.J.; Dong, J.F.; Ming, Z.; Xu, X.B.; Sun, L.N. Cold-induced endogenous nitric oxide generation plays a role in chilling tolerance of loquat fruit during postharvest storage. Postharvest Biol. Tec. 2012, 65, 5-12. [CrossRef]

20. Afrin, S.; Huang, J.J.; Luo, Z.Y. JA-mediated transcriptional regulation of secondary metabolism in medicinal plants. Sci. Bull. 2015, 60, 1062. [CrossRef]

21. Pei, T.L.; Ma, P.D.; Ding, K.; Liu, S.J.; Jia, Y.Y.; Ru, M.; Dong, J.N.; Liang, Z.S. SmJAZ8 acts as a core repressor regulating JA-induced biosynthesis of salvianolic acids and tanshinones in Salvia miltiorrhiza hairy roots. J. Exp. Bot. 2018, 69, 1663-1678. [CrossRef]

22. Ming, Q.L.; Han, T.; Li, W.C.; Zhang, Q.Y.; Zhang, H.; Zheng, C.J.; Huang, F.; Rahman, K.; Qin, L.P. Tanshinone IIA and tanshinone I production by Trichoderma atroviride D16, an endophytic fungus in Salvia miltiorrhiza. Phytomedicine 2012, 19, 330-333. [CrossRef] [PubMed]

23. Ge, X.C.; Wu, J.Y. Tanshinone production and isoprenoid pathways in Salvia miltiorrhiza hairy roots induced by $\mathrm{Ag}^{+}$and yeast elicitor. Plant Sci. 2005, 168, 487-491. [CrossRef]

24. Zhou, J.; Guo, L.P.; Zhang, J.; Zhou, S.F.; Yang, G.; Zhao, M.Q.; Huang, L.Q. Effects of $\mathrm{LaCl}_{3}$ on photosynthesis and the accumulation of tanshinones and salvianolic acids in Salvia miltiorrhiza seedlings. J. Rare Earth. 2011, 29, 494-498. [CrossRef]

25. Gao, W.; Sun, H.X.; Xiao, H.B.; Cui, G.H.; Hillwig, M.L.; Jackson, A.; Wang, X.; Shen, Y.; Zhou, N.; Zhang, L.X.; et al. Combining metabolomics and transcriptomics to characterize tanshinone biosynthesis in Salvia miltiorrhiza. BMC Genomics. 2014, 15, 73. [CrossRef] [PubMed]

26. Wang, C.H.; Zheng, L.P.; Tian, H.; Wang, J.W. Synergistic effects of ultraviolet-B and methyl jasmonate on tanshinone biosynthesis in Salvia miltiorrhiza hairy roots. J. Photochem. Photobiol. B 2016, 159, 93-100. [CrossRef] [PubMed]

27. Aremu, A.O.; Masondo, N.A.; Van Staden, J. Smoke-water stimulates secondary metabolites during in vitro seedling development in Tulbaghia species. S. Afr. J. Bot. 2014, 9, 49-52. [CrossRef]

28. Soós, V.; Sebestyén, E.; Juhász, A.; Szalai, G.; Tandori, J.; Light, M.E.; Kohout, L.; Van Staden, J.; Balázs, E. Transcriptome analysis of germinating maize kernels exposed to smoke-water and the active compound KAR1. BMC Plant Biol. 2010, 10, 236. [CrossRef] [PubMed]

29. Xu, M.J.; Dong, J.F. Synergistic action between jasmonic acid and nitric oxide in inducing matrine accumulation of Sophora flavescens suspension cells. J. Integr. Plant Biol. 2008, 50, 92-101. [CrossRef] [PubMed]

30. Xu, Y.H.; Liao, Y.C.; Zhang, Z.; Liu, J.; Sun, P.W.; Gao, Z.H.; Sui, C.; Wei, J.H. Jasmonic acid is a crucial signal transducer in heat shock induced sesquiterpene formation in Aquilaria sinensis. Sci. Rep. 2016, 6, 21843. [CrossRef] [PubMed]

31. Zhang, S.; Yan, Y.; Wang, B.; Liang, Z.; Liu, Y.; Liu, F. Selective responses of enzymes in the two parallel pathways of rosmarinic acid biosynthetic pathway to elicitors in Salvia miltiorrhiza hairy root cultures. J. Biosci. Bioeng. 2014, 117, 645-651. [CrossRef]

32. Light, M.E.; Daws, M.I.; Van Staden, J. Smoke-derived butenolide: Towards understanding its biological effects. S. Afr. J. Bot. 2009, 75, 1-7. [CrossRef]

33. Liang, W.; Chen, W.; Wu, L.; Li, S.; Qi, Q.; Cui, Y.; Liang, L.; Ye, T.; Zhang, L. Quality Evaluation and Chemical Markers Screening of Salvia miltiorrhiza Bge. (Danshen) Based on HPLC Fingerprints and HPLC-MS (n) Coupled with Chemometrics. Molecules 2017, 22, 2-16. [CrossRef] [PubMed] 
34. Li, X.; Zhang, L.; Ahammed, G.J.; Li, Z.X.; Wei, J.P.; Shen, C.; Yan, P.; Zhang, L.P.; Han, W.Y. Nitric oxide mediates brassinosteroid-induced flavonoid biosynthesis in Camellia sinensis L. J. Plant Physiol. 2017, 214, 145-151. [CrossRef] [PubMed]

Sample Availability: Samples of the compounds (tanshinone I) are available from the authors.

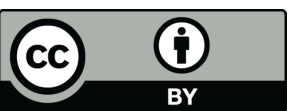

(C) 2019 by the authors. Licensee MDPI, Basel, Switzerland. This article is an open access article distributed under the terms and conditions of the Creative Commons Attribution (CC BY) license (http://creativecommons.org/licenses/by/4.0/). 